\title{
Uncovering Hidden Semantics of Set Information in Knowledge Bases
}

\author{
Shrestha Ghosh ${ }^{\mathrm{a}, *}$, Simon Razniewski ${ }^{\mathrm{a}}$, Gerhard Weikum $^{\mathrm{a}}$ \\ ${ }^{a}$ Max Planck Institute for Informatics, Saarland Informatics Campus, Saarbrücken 66123, Germany
}

\begin{abstract}
Knowledge Bases (KBs) contain a wealth of structured information about entities and predicates. This paper focuses on set-valued predicates, i.e., the relationship between an entity and a set of entities. In KBs, this information is often represented in two formats: (i) via counting predicates such as numberOfChildren and staffSize, that store aggregated integers, and (ii) via enumerating predicates such as parent0f and worksFor, that store individual set memberships. Both formats are typically complementary: unlike enumerating predicates, counting predicates do not give away individuals, but are more likely informative towards the true set size, thus this coexistence could enable interesting applications in question answering and KB curation.

In this paper we aim at uncovering this hidden knowledge. We proceed in two steps. (i) We identify set-valued predicates from a given KB predicates via statistical and embedding-based features. (ii) We link counting predicates and enumerating predicates by a combination of co-occurrence, correlation and textual relatedness metrics. We analyze $\checkmark$ the prevalence of count information in four prominent knowledge bases, and show that our linking method achieves up to $0.55 \mathrm{~F} 1$ score in set predicate identification versus $0.40 \mathrm{~F} 1$ score of a random selection, and normalized discounted gains of up to 0.84 at position 1 and 0.75 at position 3 in relevant predicate alignments. Our predicate alignments are showcased in a demonstration system available at https://counqer.mpi-inf.mpg.de/spo.
\end{abstract}

\section{Introduction}

Motivation and Problem. Knowledge bases (KBs) like Wikidata [47, DBpedia [2, Freebase [5] and YAGO 45 are important backbones for intelligent applications such as structured search, question answering and dialogue. Properly modelling and understanding the schema of such KBs, and the semantics of their predicates, is a crucial prerequisite for utilizing them. In this paper we focus on setvalued predicates, i.e., predicates which connect entities with sets of entities. Set-valued predicates typically come in two variants: (i) as enumerating predicates, which list individual objects for a given subject, and (ii) as counting predicates, which present total object counts. An example for this is shown in Fig. 1, an excerpt from Wikidata about the former US president Garfield. The predicate child, which lists individual children of Garfield is an enumerating predicate, while numberOfChildren, which gives a count of Garfield's children, is a counting predicate, and both model the same phenomenon. Set predicates can also model merely related phenomena, for instance, for a given location, the sets described via numberOf Inhabitants and birthPlace0f typically have a considerable overlap, but do not coincide.

\footnotetext{
*Corresponding author

Email addresses: ghoshs@mpi-inf.mpg.de (Shrestha Ghosh), srazniew@mpi-inf .mpg.de (Simon Razniewski), weikum@mpi-inf.mpg.de (Gerhard Weikum)
}

\section{James A. Garfield}

20th president of the United States

\begin{tabular}{|c|c|c|}
\hline \multirow[t]{4}{*}{ child } & $\hat{\nabla}$ & Eliza Garfield \\
\hline & छे & Harry Augustus Garfield \\
\hline & हิ & James Rudolph Garfield \\
\hline & छे & Abram Garfield \\
\hline number of children & छे & 7 \\
\hline
\end{tabular}

Figure 1: Example of enumerating and counting predicates in Wikidata (https://www.wikidata.org/wiki/Q34597).

Identifying set predicates and set alignments would be an important step towards a better understanding of $\mathrm{KB}$ semantics. In particular, set alignments would be beneficial for the following use cases:

(i) KB curation: Identifying gaps and inconsistencies in the $\mathrm{KB}$ and getting directives for acquiring missing pieces of knowledge (e.g., adding the 3 absent children of US president Garfield to the KB) [30].

(ii) Query formulation: Aiding users to formulate proper SPARQL queries by showing them related predicates (e.g., finding people with more than 2 children by computing the union of matches for the counting predicate 
and results from aggregating the instances of the enumerating predicate 6 .

(iii) Answer explanation: Exemplifying query results by showing key instances of queries over counting predicates (e.g., showing a few individual Turing Award winners for a query about the number of award winners).

Note that we do not advocate that all gaps between related predicates hint at errors or incompleteness that require actions. Scope of a KB, (non-)notability of entities, or privacy considerations may well motivate that certain gaps should not be filled, and temporal semantics may add subtleties (e.g., one predicate only counting current employees, the other storing also historical members).

While there is a rich body of research on ontology alignment and schema matching [36, 11, 40, 20, 44, 49, 32, 4], these works typically focus on identifying perfectly matching pairs of predicates with the same or largely overlapping values. This situation differs from our setting where the integer values of counting predicates and the cardinalities of enumerating predicates modelling the same or related phenomenon rarely match perfectly. Properly identifying set predicates and set alignments in knowledge bases is also difficult for other reasons: (i) KBs contain a large number of predicates, often with uninformative names and without coherent type signature, thus making the identification of set predicates and their alignments challenging. (ii) Enumerating predicates are often incomplete (like Garfield's children) and counting predicates may be approximate estimates only (like number of inhabitants); so cardinalities do not match count values, yet the predicates should be linked in order to couple them for future KB completion, consistency assessment and other use cases listed above.

Approach and Contribution. This paper presents CounQER (for "Counting Quantifiers and Entity-valued PRedicates"), the first comprehensive methodology towards identifying and linking set predicates in KBs. CounQER is judiciously designed to identify set predicates in noisy and incomplete web-scale KBs such as Wikidata, DBpedia and Freebase. It operates in two stages: In the first stage, supervised classification combining linguistic and statistical features is used to identify enumerating and counting predicates. In the second stage, a set of statistical co-occurrence and correlation measures is used in order to link the set predicates.

Our salient original contributions are:

1. We introduce the notion of set predicates, its variants, and highlight the benefits that can be derived from identifying their alignments.

2. We present a two-stage methodology for (i) predicting the counting and enumerating predicates via supervised classification and, (ii) ranking set predicates

${ }^{1}$ Example query for people with $>2$ children: http://tinyurl. $\mathrm{com} / \mathrm{y} 4 \mathrm{hemdvc}$ of one variant aligned to the other variant via statistical and lexical metrics.

3. We demonstrate the practical viability of our approach by extensive experiments on four KBs: Wikidata, Freebase, and two variants of DBpedia, .

4. We publish results of our alignment methodology for these KBs at https://tinyurl.com/y2ka4kfu which contains 264 alignments from DBpedia mappingbased KB, 3703 alignments from the DBpedia raw $\mathrm{KB}, 25$ alignments from the Wikidata-truthy KB, 274 alignments from the Freebase KB and, an interactive demo QA system at https://counqer.mpi-irff. $\mathrm{mpg} \cdot \mathrm{de} / \mathrm{spo}$.

\section{Related Work}

Schema and ontology alignment. Schema alignment is a classic problem in data integration [36. For ontologies and on the semantic web, added complexity comes from taxonomies and ontological constraints [11, 40]. Approaches to ontology alignment include BLOOMS [20] and PARIS 44, voting-based aggregation 49, probabilistic frameworks 32 , or methods for the alignment of multicultural data 4. These methods typically rely on a combination of lexical, structural, constraint and instance based information. Some works have also investigated subset relations [22], yet still focusing only on entity-entity relations. The most important venue in the field of ontology alignment is the long-running Ontology Matching workshop series 41 along with its attached challenges [1]. Our setting, where enumerations need to be aligned with counts, is atypical in ontology alignment and has not received prior attention.

Set information in logics and KBs. Modelling count information has a history in qualifying number restrictions [18 and role restrictions in description logics [8]. In the OWL standard [28], count information on relations can be expressed via cardinality assertions. The second statement in Fig. 1, for instance, could be expressed as

ClassAssertion (ObjectExactCardinality (7 :child) :Garfield)

OWL furthermore also supports lower bounds, e.g., that a certain person has at least two children, and upper bounds, e.g., that a certain car has at most five seats. The added expressiveness from counting quantifiers typically comes at a complexity tradeoff, no matter whether they are part of the ontology language [17, 7], or only of the query language [13, 33, 10, especially as they introduce negation ("Companies that are owned by zero other companies"). Despite several methods for automatically learning logical axioms and patterns [23, 15], we are not aware of attempts to learn set relatedness.

Numeric and set information in KBs, QA and IE. Popular knowledge bases contain considerable numeric information. Research has focused on detecting errors and 
outliers in such information [50], and in organizing and annotating measurement units [31, 43.

Set information is important for question answering. For instance, in 30 it is reported that between $5 \%$ and $10 \%$ of questions in popular TREC QA datasets concern counts. This information need, although acknowledged by QA systems, is so far not dealt with in a principled manner. AQQU [3, for instance, includes a special translation for questions starting with "How many?". The Google search engine similarly answers count keyword queries for popular entities like "How many children Angelina Jolie" with both counts and instances, yet there is no discernible pattern concerning which queries evoke this behaviour, and it occurs only for very few highly popular ones.

When concerned with numeric information, textual information extraction traditionally focused on temporal information [26] and measures 39. Recently also counting information extraction has received attention, e.g., from sentences like "The LoTR series consists of three books" 29 30 . Such information can then be used to assess and improve $\mathrm{KB}$ completeness [38, 37]. In the present work, we investigate the identification and alignment of set predicates in knowledge bases itself, i.e., without external text sources.

KB recall information. Recall is an important dimension of KB quality, with impact on downstream use cases 34. Unlike precision, it cannot be easily evaluated by sampling. Existing approaches to $\mathrm{KB}$ recall estimation can be grouped into three families. The first are approaches based on statistical patterns in the data, e.g., sample overlap [27, digit-distributions 42], or association rules 14. The second are relative approaches, i.e., where recall expectations are collected from related entities [19, 42]. The third are text-extraction based approaches mentioned above. The present work complements these approaches with the exploration of predicate interrelations among set predicates.

\section{Problem Definition}

Let $P$ be a set of predicates. A knowledge base (KB) is a set of triples $(s, p, o)$, where $p \in P, s$ is an entity, and $o$ is either an entity or a literal. For the remainder of this paper we assume that each triple $(s, p, o)$ with an entity as object also exists in its inverse form $\left(o, p^{-1}, s\right)$ in each $\mathrm{KB}$, thus the following elaborations need to consider only one direction.

The foundational concept for this work is that of a set predicate.

Definition 1 (Set Predicat $\AA^{2}$ ). A predicate which conceptually models the relation between an entity and a set of

${ }^{2}$ We emphasize that set predicate refers to the intended semantics of the modeller, not to be mixed with the capabilities of the modelling language. In particular, unlike SQL, the RDF data model does not know a $S E T$ datatype, but can capture sets via multiple triples sharing subject or object. entities is a set predicate.

Set predicates can be expressed in KBs in two variants: Via binary predicates that enumerate individual set members, and via counting predicates that abstract away from individuals, and store aggregate counts only.

Definition 2 (Enumerating Predicate). A set predicate that models sets via binary membership statements is called an enumerating predicate.

We denote the set of enumerating predicates as $E$.

Definition 3 (Counting Predicate). A set predicate whose values represent counts of entities modelled in the considered $\mathrm{KB}$ is called a counting predicate.

Entity counts necessarily are integers, yet KB predicates can contain integers that represent a variety of other concepts, for instance identifiers or measures like length and weight.

Following these definitions, the predicates child and numberOfChildren in Fig. 1 are set predicates, the former an enumerating predicate, the latter a counting predicate. Other examples are worksAt ${ }^{-1}$ and author0f, which frequently enough take several values for a subject. This is in contrast to predicates of a functional or quasi-functional nature, such as bornIn and mother, which predominantly take a single object, and hence, where counts are uncommon and rarely informative. Yet the threshold for enumerating predicates is imprecise, for instance, the predicate citizenOf is predominently functional, but some entities still have multiple citizenships which are conceivably countable.

Other examples of counting predicates are population, numberOfStudents and airlineDestinations. The distinction between counting predicates and measurement predicates like riverLength and revenue is quite crisp, since measurements usually come with units ( $\mathrm{km}, €$, etc.) and can take fractional values $(1.7 \mathrm{~km})$ while entity counts cannot. Our definition is phrased to also exclude some predicates taking integer values, like episodeNumber (not a count, but a sequential number assigned to an episode of a TV series) and floorCount (a count, but not of something commonly considered as entities). Thus, integer values are a necessary but not a sufficient condition for being a count predicate.

We summarize our first problem as follows.

Problem 1 (Set predicate identification). Given a $\mathrm{KB}$ with a predicate set $P$, identify the set of enumerating predicates, $E$, and the set of counting predicates, $C$.

Note that the above definitions are conceptual only. Functionalities computed over actual KBs are unreliable due to incompleteness, errors, and redundancies, and common KBs do not have a entity-count datatype. Thus, in later sections we will develop supervised classifiers for identifying both kinds of set predicates. 
Let us now turn to the relation between set predicates. Set-relatedness refers to the (ideal) amount of overlap that two set predicates have on a per-subject basis. For instance, in a perfect $\mathrm{KB}$, child and numberOfChildren describe exactly the same set of objects per subject, once via a listing of names, ones via the aggregate count.

In turn, the predicates population and bornIn ${ }^{-1}$ do not describe the same sets, but would typically exhibit a significant overlap (many people live in the same place they are born in, though neither entails the other). On the other hand, population and headquarterLocation ${ }^{-1}$ are not set related. Although population sizes and the number of company headquarters in a place are correlated numbers, the described entities do not overlap at all, instead, are even of distinct types (person and company).

Conceptually, set relatedness between two predicates can therefore be computed as an across-subjects aggregated set overlap measure, with perfect matches being the strongest relatedness. Note that this definition is strictly conceptual, since in actual KBs, counting predicates do not give away which actual entities they count.

Problem 2 (Set predicate alignment). Given sets of enumerating predicates $E$ and counting predicates $C$, for each set predicate $p \in E \cup C$, rank the predicates from the other set by their set-relatedness.

Note that the above definitions of set-relatedness are conceptual definitions. In practice, KBs do not give access to the entities counted by counting predicates, instead one only sees aggregate counts. To quantify and qualify setrelatedness, in the following sections, we will thus build a set of unsupervised alignment heuristics.

\section{Design Space and Architecture}

Design space. Our goal is to develop a robust set predicate identification and linking methodology, that, with limited supervision, can work across different KBs.

If knowledge bases were clean, set predicate identification could solely rely on relation functionality and datatypes. As this is not the case in practice [51, 50, 52, we instead propose to approach set predicate identification via a supervised classification framework that combines a diverse set of textual, datatype, and statistical features. Schema alignment can also in principle be approached via hand-crafted rules, heuristic alignment metrics, or supervised learning. Due to the particularities of individual predicates and KBs (most set predicates have only very few good alignments), to avoid overfitting, we opt here for a set of heuristic alignment metrics, which we design in order to capture various desiderata of meaningful alignments, and combine them in an ensemble metric.

Architecture. Following the above considerations, we split our CounQER methodology in two steps: (i) supervised predicate classification, and, (ii) heuristic predicate alignment (see Fig. 2).

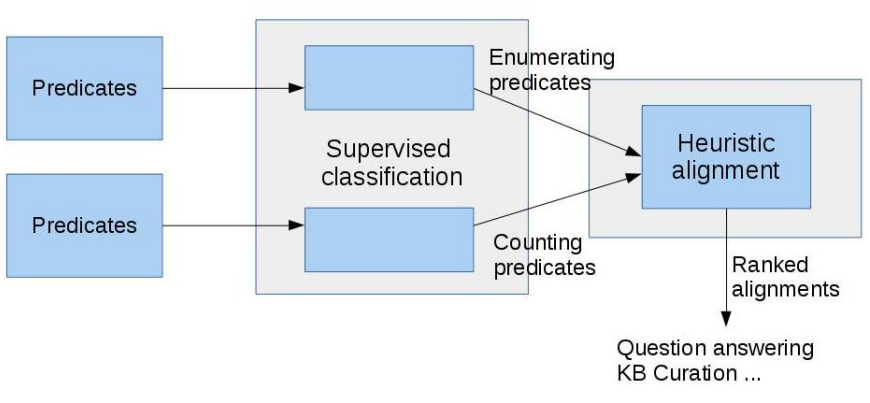

Figure 2: Architecture of the CounQER approach.

In the first phase, supervised predicate classification, we use two classifiers to predict the two set variants, namely, enumerating and counting predicates. We rely on a family of features, most importantly, (i) set-related textual features, extracted from a background corpus, (ii) type information about the domain and range of the predicates, and (iii) statistical features about the number of objects per subject at different percentiles.

In the second phase, heuristic predicate alignment, we identify related counting and enumerating predicates using (i) set predicate co-occurrence information, (ii) set predicate value distribution, and (iii) linguistic relatedness. By assigning each pair of enumerating and counting a relatedness score, we can rank related predicates accordingly. While we evaluate the heuristics on labelled data, they are highly complementary, and thus, the choice of the heuristic to be used can be adapted to particular use cases.

KB assumptions. Our approach is designed to work on a variety of knowledge bases, without requiring strong assumptions on their internal structure. Fulfilment of the following features is desirable, though not essential: (i) High-level categories/classes for entities, in particular Person, Place, Organisation, Event and Work. Where these are not available, we utilize links to Wikidata to extract them. (ii) High-level datatypes, in particular float, int and date. Where these are not available, we utilize standard parsers and simple heuristics, such as that numbers between 1900 and 2020 are likely dates. (iii) human-readable labels for properties, with spaces or in camel case notation. Where these are not available, we deactivate corresponding linguistic features.

\section{Set Predicate Identification}

\subsection{Enumerating Predicates}

As stated in Section. 3, if KBs were clean, functionality (\#triples per subject) would be the criterion for identifying enumerating predicates.

Yet actual KBs contain a considerable amount of noise, are incomplete, and blur functionality by redundancies (e.g., listing both the birth city and country of a person under birthPlace). In CounQER, we thus rely on supervised classification, where functionality is only one among 
several features towards enumerating predicate identification.

\section{a. Textual features}

Where KBs use human-readable predicate names, a basic sanity check for enumerating predicates is to verify whether in human language, the predicate name is used both in singular and plural.

1. Plural-singular ratio: For each predicate, we apply a heuristic to we generate its plural/singular form. First we identify the last noun in the predicate label using the Python nltk package and then we use the Python inflect library to identify its form (singular/plural) and convert it to the other (plural/singular). We then compute the text frequency ratio based on the Bing API, obtaining, for instance, for $\frac{\mid \text { children } \mid}{\mid \text { child } \mid}$ a ratio of $\frac{128.000 .000}{87.000 .000}=1.47$, while for birthplace, the ratio is $\frac{\mid \text { birthplaces } \mid}{\mid \text { birthplace } \mid}=\frac{1.550 .000}{21.000 .000}$, a ratio of 0.08 .

\section{b. Type information}

Subject and object types may explain the applicability of other features, and certain types of objects may more naturally be counted than others, and certain subjects may more frequently come with set predicates than others. To avoid overfitting and ensure compatibility across KBs, in this work we only consider five frequent and general classes, \{Person, Place, Organisation, Event and Work\}, which we use to capture the domain and range type of a predicate. Further details follow in Sec. 7.2.

2. Predicate domain: We encode the most frequent class of a predicate domain via binary features per class, including a 6th class for other.

3. Predicate range: We encode the class of predicate range in binary variables (same as in predicate domain).

\section{c. KB statistics}

KB statistics instantiate the observed functionality. As functionality may be blurred by outliers or a long tail of single-valued subjects, we input various datapoints in order to increase resilience of the measure. We also include basic information on datatypes.

4. Mean, maximum, minimum, 10th and 90th percentile number of objects per subject (functionality): These features describe the number of objects a predicate takes per subject, with mean and percentiles giving resilience against rare outliers. For example, occupation in Wikidata-truthy KB has a mean of 1.3 , maximum of 30 , minimum of 1,10 th percentile of 1 and 90th percentile of 2 . The predicate place0f Birth in Wikidata-truthy KB has a maximum of 6 objects per subjects and 1 object per subject for the other features - minimum, mean, 10th and 90th percentile.
5. Datatype distribution: The fraction of triples of a predicate whose objects are of datatype entity. For instance, both the predicates occupation and placeofBirth take entities for $99 \%$ of the triples in Wikidatatruthy KB.

These features are then used for a binary classifier.

\subsection{Counting Predicates}

As per our conceptual definition, counting predicates are distinguished by having entity-count as their datatype. As none of the KBs investigated in this paper records such a datatype, we have to use various heuristic towards identifying counting predicates. An important necessary condition are integer values, yet these alone are not sufficient. We utilize the following classes of features.

\section{a. Textual features}

1. Plural-singular ratio: This feature captures the plu$\mathrm{ral} /$ singular ratio of a predicate obtained exactly as for enumerating predicates.

\section{b. Type information}

2. Predicate domain: We identify the domain of the predicates by tracing the class of the predicates to one of the most general classes in the type heirarchy, \{Place, Person, Organization, Event, Work $\}$. Each of the domain class is encoded as a binary variable in the classifier.

\section{c. KB statistics}

3. Datatype distribution: We calculate the fraction of triples of a predicate taking integer values over the total number of triples of that predicate. For instance, the predicate numberOfEpisodes in the DBpedia mapping-based KB takes only integer values, whereas episodeNumber in the DBpedia raw KB takes integer values for $96 \%$ of the triples.

4. Mean, maximum, minimum, 10th and 90th percentile of count value: These features describe the actual integer value of the predicate, e.g., the mean for numberOfEpisodes (DBpedia mapping-based KB) is 106 , the maximum is 90015 , the minimum is 0 , the 10th percentile is 6 and the 90th percentile is 156 .

5. Mean, maximum, minimum, 10th and 90th percentile of the number of objects per subject (functionality): These features describe the number of integer valued triples per subject.

For example, the mean numberOfEpisodes (DBpediamapping-based KB) a subject takes is 1 , the maximum is 8 , the minimum, the 10 th percentile and the 90 th percentile all are 1 , i.e., most subjects have only one fact containing this predicate. In contrast, an ordinal integer predicate like episodeNumber (DBpedia raw $\mathrm{KB}$ ) has the following statistics - mean 32 , maximum 975, minimum 1, 10th percentile 6 and 
90th percentile 66 . This odd behaviour is exhibited because the article page lists all or a subset of the episode numbers in a series 3

\section{Heuristic Predicate Alignment}

The output of the previous stage are the enumerating predicates $E$ and the counting predicates $C$. The task of this stage is to find for each predicate in $E \cup C$ the most set-related predicates from the other set. As this task may to some extent be KB-specific, we approach it via a set of unsupervised ranking metrics. We introduce three families of metrics for predicate pairs: (a) set predicate co-occurrence, based on the number of subjects for which $e \in E$ and $c \in C$ co-occur, (b) set predicate value distribution, based on the relation between the number of objects in $e$ and the value of $c$ for co-occurring subjects, and (c) set predicate linguistic similarity which measures the relatedness between the labels of the set predicates $e$ and $c$. Here, we illustrate each heuristic using the enumerating predicate gold ${ }^{-1}$, which links an entity (sportsperson) to the winning sports events, and the counting predicate singlestitles, which gives the count of singles titles of a tennis player.

A. Set Predicate Co-occurrence. Our first family of heuristics ranks predicates by their co-occurrence. Cooccurrence is an indication towards topical relatedness, and we propose various measures that capture absolute and relative co-occurrence frequencies.

1. Absolute $(e, c)$ : The number of subjects which have triples with both $e$ and $c$ set predicates. For instance,

$$
\text { Absolute(singlestitles, gold } \left.{ }^{-1}\right)=64 \text {. }
$$

2. $\operatorname{Jaccard}(e, c)$ : The ratio of the absolute number of subjects for which $e$ and $c$ co-occur, i.e., Absolute $(e, c)$ divided by the union of subjects which take either $e$ or $c$ or both. For instance,

$$
\operatorname{Jaccard}\left(\text { singlestitles, gold }{ }^{-1}\right)=0.006 \text {. }
$$

3. Conditional $(e, c)$ : Co-occurrence can also be expressed as a conditional probability, i.e., the ratio of the absolute value, Absolute $(e, c)$, to the number of subjects which take either $c$ or $e$. For our given example,

$$
\text { Conditional }_{E}\left(\text { singlestitles, gold } \mathrm{d}^{-1}\right)
$$

$$
=0.011 \text {. }
$$

with respect to subjects only taking the predicate gold $d^{-1}$ and,

$$
\text { Conditional }_{C}\left(\text { singlestitles,gold }{ }^{-1}\right)=0.015 \text {. }
$$

${ }^{3}$ DBpedia subjects with count of episodenumber facts https:// tinyurl.com/dbpedia-raw-episodenumber with respect to subjects only taking the predicate singlestitles. This implies that if a given subject has the predicate singlestitles, it is more likely that the subject also has the predicate gold $\mathrm{d}^{-1}$ than the other way around.

4. PairwiseMutualInformation $(e, c)$ or (P'wiseMI): The $\log$ of the ratio of the joint distribution of $e$ and $c$ to their individual distributions.

$$
\begin{aligned}
& \operatorname{PMI}(e, c)= \\
& \log _{2} \frac{|\{s \mid s \in\langle s, e, \cdot\rangle ; s \in\langle s, c, \cdot\rangle\}| \times|\{s \mid s \in\langle s, \cdot, \cdot\rangle\}|}{|\{s \mid s \in\langle s, e, \cdot\rangle\}| \times|\{s \mid s \in\langle s, c, \cdot\rangle\}|} \\
& P M I\left(\text { singlestitles, gold }{ }^{-1}\right)=-5.2 .
\end{aligned}
$$

which implies that the two predicates are less likely to co-occur than expected from their individual occurrences. In general, this metric ranges between $-\infty$ and $\min (-\log p(e),-\log p(c))$. The lower bound is reached when the pair $(e, c)$ does not co-occur for any subject and the upper bound is reached when either $e$ always co-occurs with $c$ or vice-versa.

B. Set Predicate Value Distribution. Co-occurrence is important but can nonetheless be spurious, e.g., when many sports teams have both the predicates stadiumSize and coach0f. A possibly even stronger indicator for set relatedness is a match or correlation in values, i.e., if across subjects, the number of values for the enumerating predicate, and the count stored in the counting predicate, coincide, or correlate. We propose three variants: (5) To count the number of exact matches, and in (6) and (7) two relaxed metrics that look for correlation and percentile similarity.

5. PerfectMatchRatio $(e, c)$ : The ratio of subjects where the number of objects in $e$ exactly matches the value in $c$ to the number of subjects which takes both $e$ and $c$ predicates. For example,

$$
\left.P^{\prime} \text { fectMR(singlestitles, } \text { gold }^{-1}\right)=0.125 \text {. }
$$

6. Correlation $(e, c)$ : The Pearson correlation between the size of objects of $e$ and the value of $c$ for all subjects in which they co-occur. For the above predicate pair,

$$
\text { Correlation }\left(\text { singlestitles,gold }{ }^{-1}\right)=0.724 \text {. }
$$

7. PercentileValueMatch $(e, c)$ : A softer score than a perfect match ratio (B 5), for matching the 90 th percentile value of the number of objects that $e$ takes per subject with the 90th percentile value of the $c$, such that the closer the value is to 1 the better the 
alignment. Let $O_{c}$ and $O_{e}$ denote the distribution of the values and the \#objects per subject, respectively.

$$
\begin{aligned}
& \text { P'tileVM(e,c) }= \\
& \qquad \operatorname{Min}\left(\frac{p^{\prime} \text { tile }\left(O_{e}, 90\right)}{p^{\prime} \text { tile }\left(O_{c}, 90\right)}, \frac{p^{\prime} \text { tile }\left(O_{c}, 90\right)}{p^{\prime} \text { tile }\left(O_{e}, 90\right)}\right)
\end{aligned}
$$

P'tileVM(singlestitles, gold $\left.^{-1}\right)=0.333$.

C. Linguistic Similarity. Besides co-occurence, also correlations can be spurious. For instance, population and headquarterLocation ${ }^{-1}$ are well correlated (bigger cities host more companies), but nonetheless, they refer to completely different kinds of entities (persons vs. companies). Our third family of heuristics thus looks at topical relatedness.

8. CosineSimilarity $(e, c)$ measures the cosine of the angles between the average of the sets of word vectors of the labels of $e$ and $c$ obtained from pre-trained Glove embeddings 35 using the Python Gensim library. Wikidata predicate labels are already individual words, for DBpedia and Freebase we split the predicates at capitalization and punctuation, respectively. For example, headquarterLocation ${ }^{-1}$ becomes \{headquarter, location\} and race_count becomes $\{$ race, count $\}$.

$$
\text { CosineSim(titles, gold } \left.\mathrm{d}^{-1}\right)=0.318
$$

Out of vocabulary words, like singlestitles, lead to an empty word list. Similarity with an empty word list is assigned a score of zero as follows.

$$
\text { CosineSim(singlestitles, gold } \left.\mathrm{d}^{-1}\right)=0 \text {. }
$$

D. Alignment Summary. In the following experiments we evaluate the alignment heuristics individually against ground truth annotations on the NDCG [21] score. In this way, we can discover the best performing heuristic, and with enough training data, could even perform ensemble learning. Yet as reliance on a single heuristic would be brittle, and ensemble learning requires larger evaluation data, as a robust best-effort, we propose here to retain from each of the three families of heuristics the best performing one, and merge their scores by using averaging.

\section{Experiments}

\subsection{KBs used}

We use four popular general purpose KBs: (i) DBpedia raw extraction [2], (ii) DBpedia mapping-based extraction $^{4}\left[24\right.$, (iii) Wikidata truthy ${ }^{5}$ 47] and (iv) Freebase ${ }^{6}[5]$. We analyze each $\mathrm{KB}$ in terms of predicate coverage.

\footnotetext{
${ }^{4}$ We used DBpedia version 2016-10 for both extraction.

${ }^{5} \mathrm{We}$ used the version as of the Oct 2018.

${ }^{6}$ We used the version as of 2019 July.
}

\begin{tabular}{|c|c|c|}
\hline KB & All & Frequent \\
\hline DBP-raw & 73,234 & 16,635 \\
DBP-map & 2,008 & 1,670 \\
WD-truthy & 6,111 & 4,067 \\
Freebase & 799,807 & 13,872 \\
$Y A G O$ & $(\mathbf{7 9})$ & $(\mathbf{7 9})$ \\
\hline
\end{tabular}

Table 1: Total number of KB predicates (direct + inverse) and most frequent ones.

1. DBpedia raw (52.6M triples): All predicate-value pairs present in the infoboxes of English Wikipedia article pages.

2. DBpedia mapping-based (29M triples): A cleaner infobox dataset where predicates were manually mapped to a human-generated ontology. Unmapped predicates and type violating triples are discarded.

3. Wikidata truthy (210.3M triples): Simple triple export of Wikidata that ignores some advanced features such as qualifiers and deprecated ranks.

4. Freebase (1B triples): The tuple store available as an RDF dump at https://developers.google. com/freebase

We also analysed YAGO [45] (1.1B triples), a WordNetaligned and sanitized harvest of Wikipedia infobox statements, containing only 76 distinct predicates. By manual inspection we found several enumerating predicates, like hasChild and isCitizenOf, but only one counting predicate, numberOfPeople and therefore refrained from further processing of this KB.

On adding inverse triples, i.e., adding $\left(o, p^{-1}, s\right)$ for every $(s, p, o)$ where $o$ is an entity, the size of DBpediaraw increased by $7.6 \mathrm{M}$, DBpedia-map by $18 \mathrm{M}$, Wikidata by $101.1 \mathrm{M}$ and Freebase by $442.1 \mathrm{M}$.

To reduce noisy data we use predicates which appear in at least 50 triples. In Table 1 we show the number of predicates that remain after filtering all infrequent predicates. It is evident that the cleaner KBs like Wikidata and DBpedia mapping-based KB have better predicate representation. Freebase and DBpedia raw KBs are noisier with a very long tail of less frequently occurring predicates.

\subsection{Preprocessing}

Predicate statistics computation. Given a KB of SPO triples, we generate the descriptive statistics of the KB predicates including (i) the datatype distribution fraction of the triples of a predicate which take integer, float, date, entity and comma-separated string values, (ii) the mean, maximum, minimum, 10th and 90th percentile of the integer values that a predicate takes, (iii) the mean, maximum, minimum, 10th and 90th percentile of the number of entities per subject of a predicate and, (iv) the mean, maximum, minimum, 10th and 90th percentile of the number of integer values per subject of a predicate. We identify comma-separated string values as a datatype in order to 
handle noisy representations, especially in DBPedia-raw, where object sets are often captured in a single string with comma separation (e.g., "children: Mary, John, Susan").

Type information. We then proceed to find the predicate domain and range. To maintain uniformity across KBs we trace the type to one of the more general classes in the type hierarchy, \{Place, Person, Organization, Event, Work\}, with the default fallback class for entities being Thing and non-entities being Literal. The fallback classes capture long-tailed classes and string objects, which have no class information.

We sampled 100 subjects and 100 objects for each predicate and selected the majority class in each set as the domain and range of the predicate. Across all four KBs, the (micro-average) coverage of the predicate domain by the classes are $\{$ Place: $18 \%$, Person: $23 \%$, Organization: 14\%, Event: $4.75 \%$, Work: $17.25 \%$, Thing: $23 \%\}$ and, for predicate range, $\{$ Place: $18.25 \%$, Person: $20 \%$, Organization: $15.25 \%$, Event: $1.25 \%$, Work: $17 \%$, Thing: $1.75 \%$, Literal: $26.5 \%$.

Linguistic features. The frequency of occurrence of a predicate in the web in singular and plural form is determined from the total estimated web search matches returned by the Bing custom search API 7 For inverse predicates, we reuse the predicate labels of their forward form for getting the textual features for the classifiers and the linguistic similarity measure for the alignment heuristics.

\subsection{Training and evaluation data}

We prepared the data for the classification step by employing crowd workers to annotate 400 randomly selected predicates for enumerating predicates and 400 for counting predicates from the four KBs - taking 100 from each KB. The annotation task comprised a predicate and five sample subject-object pairs with options to select if the predicate was likely a set predicate (enumerating or counting).

An example question for annotating counting predicates is given below.

Q: Based on the following facts, decide whether the relation gives a count of unique entities.

The Herald (Sharon)

H.O.W. Journal

L'Officiel

The Music Scene (magazine)

Pipe Dream (newspaper) circulation 15715

circulation 4000

circulation 101719

circulation 25000

circulation 7000

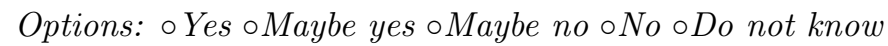

For enumerating predicate annotation we used the following question format.

Q: Based on the following facts, decide whether the relation enumerates entities.

\footnotetext{
${ }^{7}$ https://azure.microsoft.com/en-us/services/cognitiveservices/bing-custom-search/
}

\begin{tabular}{|lcc|}
\hline KB & Positive samples & Negative Samples \\
\hline DBP-raw & 16 & 62 \\
DBP-map & 9 & 72 \\
WD-truthy & 7 & 87 \\
Freebase & 7 & 85 \\
Total & 39 & 306 \\
\hline
\end{tabular}

Table 2: Distribution of counting classifier training samples across KBs.

\begin{tabular}{|lcc|}
\hline KB & Positive samples & Negative Samples \\
\hline DBP-raw & 33 & 53 \\
DBP-map & 27 & 58 \\
WD-truthy & 27 & 55 \\
Freebase & 46 & 29 \\
Total & 133 & 195 \\
\hline
\end{tabular}

Table 3: Distribution of enumerating classifier training samples across KBs.

A Low Down Dirty Shame Bye Bye Brazil

Heaven Knows, Mr. Allison

Surviving Paradise

I'll Come Running Back to You producer Mike Chapman producer Luiz Carlos Barreto producer Eugene Frenke producer Kamshad Kooshan Options: ○ Yes oMaybe yes o Maybe no $\circ$ No ○Do not know

We collected three judgements per predicate, i.e., a total of 2400 annotations ( 2 variants of set predicates $\times 4$ KBs $\times 100$ predicates $\times 3$ judgments $)$. The options in the annotation task are graded. We translated the labels into numeric scores \{Yes: 1, Maybe yes: 0.75, Do not know: 0.5, Maybe no: 0.25, No: 0\}, with the final label being the average of all judgments. Concerning annotator agreement, we found the pooled standard deviation of the scores per predicate to be 0.41 . We only keep rows with a clear polarity, i.e., rows with average score outside the interval $(0.4,0.6)$, effectively excluding rows averaging around the option Do not know. The labels of the remaining rows are then translated into binary 0-1-judgments. Of the counting predicate rows, $86.25 \%$ showed a clear polarity, of the enumerating predicate rows, $82 \%$. Thus, we obtained our training data, with 39 positive and 306 negative data points for the counting classifier and, 133 positive and 195 negative data points for the enumerating classifier.

We can conclude from Table 2 that in general, KBs contain comparably few counting predicates, which also contributes to the low precision score of the counting classifier. From the numbers in Table 3 , we observe that enumerating predicates have a comparably higher occurrence.

For the alignment step, evaluation data was prepared by collecting relevance judgements from crowd workers. We randomly chose 300 enumerating and 300 counting predicates as returned by our classifiers. As co-occurring predicate pairs have a long tail of infrequent pairs that due to small sample size might lead to spurious heuristics scores, we set a threshold on the absolute co-occurrences for the alignments. Of all co-occurring pairs we consider 
those which co-occur for at least 50 subjects for annotation, evaluation and final ranking purposes. We then created the set of top-3 counting predicates returned by all the alignment heuristics for each enumerating predicate, so that for each enumerating predicate we had up to 27 counting predicates as candidates. Note that the alignment is KB-specific, so we return top-3 predicates from the same KB to which the enumerating predicate belongs.

We repeated the step with the counting predicates, this time returning the top-3 enumerating predicates for each counting predicate. On an average, there were 5 candidates for each set predicate in the enumerating and counting case. The annotation task asked each worker to judge the topical relatedness of a pair of set predicates (an enumerating and a counting predicate) and the degree of completeness based on the integer value of the counting predicate and the entities covered by the enumerating predicate with respect to a subject. An example task where the system returns a counting predicate is as follows.

$\begin{array}{lllr}\begin{array}{l}\text { Subject } \\ \text { Query }\end{array} & \text { Predicate } & \text { Object } \\ \text { Univ. of California, L.A. } & \text { institution }{ }^{-1} & \text { Thomas Sowell, } \\ & & \text { Harold Demsetz } \\ & & \text {..(5 in total) }\end{array}$

\section{Result}

Univ. of California, L.A. faculty size 4016

We ask the following two questions.

\section{Topical relatedness of institution $^{-1}$ to faculty size} is:

Options: ○ High ○ Moderate ○ Low ○ None.

2. Enumeration of the objects in the query is:

Options: ○ Complete $\circ$ Incomplete ○ Unrelated.

The task in the opposite direction is designed in a similar fashion with the query containing a counting fact and the result, an enumerating fact with the set of objects.

For this task also we collected three judgements per predicate pair in either direction. We again used a graded relevance system by calculating a mean score of the two responses where, the grades for topical relatedness are \{High: 1, Moderate: 0.67, Low: 0.33, None: 0\} and for the completeness of enumeration we have \{Complete: 1, Incomplete: 0.5, Unrelated: 0$\}$. Thus the graded relevance score ( 1 being the highest and 0 being the lowest) is calculated by mapping the responses to their grades and averaging over all responses. Concerning agreement, the pooled standard deviation of responses across pairs was 0.3 for topical relatedness and 0.46 for completeness of enumeration.

\subsection{Classifier models}

We model our classifiers on logistic regression as well as neural networks. However, due to small dataset size, and our interest in interpretable insights, we focus on multiple logistic regression models. We consider a standard logistic regression model, a logistic regression model with a weakly informative default prior 16, a Lasso regularized logistic

\begin{tabular}{|c|ccc|}
\hline Model & Recall & Precision & F1 \\
\hline Random & 12.8 & 12.8 & 12.8 \\
Logistic & 51.2 & 19.0 & 27.7 \\
Prior & 48.7 & 20.2 & 28.5 \\
Lasso & $\mathbf{7 1 . 7}$ & $\mathbf{2 3 . 3}$ & $\mathbf{3 5 . 1}$ \\
Neural & 35.8 & 20.8 & 26.3 \\
\hline
\end{tabular}

Table 4: Performance (precision, recall and F1) of the counting predicate classifier models.

\begin{tabular}{|l|ccc|}
\hline Model & Recall & Precision & F1 \\
\hline Random & 40.6 & 40.6 & 40.6 \\
Logistic & $\mathbf{5 5 . 6}$ & 51.7 & 53.5 \\
Prior & $\mathbf{5 5 . 6}$ & 51.0 & 53.5 \\
Lasso & 51.1 & $\mathbf{5 9 . 6}$ & $\mathbf{5 5 . 0}$ \\
Neural & 53.0 & 49.6 & 51.2 \\
\hline
\end{tabular}

Table 5: Performance (precision, recall and F1) of the enumerating predicate classifier models.

regression [46] and a neural network composed of a hidden layer of size three and sigmoid activation function. Due to the small training set we use Leave-One-Out cross validation to obtain our model performance scores.

All models are compared against a random baseline modelled on the input distribution, i.e., predicting labels at random, with probabilities proportional to label frequency in the training data.

\subsection{Results}

a. Classifier model selection. The results of the classifier models are in Tables 4 and 5 . As one can see, the Lasso regularized model performs the best for counting predicates with an F1 score of 35.1 , which is significantly better than the random model which has an F1 score of 12.8. We observe that the counting classifier models in general have lower precision scores, but higher recall. The scores of the random model are computed from the training data distribution of counting predicates which contain 39 positive and 306 negative datapoints. Note that the number of datapoints is less than the initial selection of 400 datapoints since, as explained in the previous section, we remove datapoints with divided agreements. We use the Lasso regularized model to classify the counting predicates.

In the enumerating predicate scenario also, the Lasso regularized model has an overall highest performance with an F1 score of 55. Here too, the random classifier performance depends on the distribution of training data which has 133 positive and 195 negative datapoints, giving an F1 score of 40.6. We use the Lasso regularized model for predicting the enumerating predicates. The comparable recall and precision scores of the enumerating predicate classifier can be attributed to the almost equal class distribution in the training data, which is not the case for counting predicates. 


\begin{tabular}{|c|ccc|}
\hline KB & Input & Output & Filtered \\
\hline DBP-raw & 16,635 & 4,090 & $4,090(24.5 \%)$ \\
DBP-map & 1,670 & 308 & $308(18.4 \%)$ \\
WD-truthy & 4,067 & 216 & $203(4.9 \%)$ \\
Freebase & 13,872 & 7,752 & $7,614(54.8 \%)$ \\
\hline Total & 36,244 & 12,366 & $12,215(33.7 \%)$ \\
\hline
\end{tabular}

Table 6: Predicted enumerating predicates across different KBs, where Input is all $\mathrm{KB}$ predicates (direct + inverse), Output is from the classifier prediction and Filtered the number of predicates remaining after removing predicates related to IDs and codes.

\begin{tabular}{|c|ccc|}
\hline KB & Input & Output & Filtered \\
\hline DBP-raw & 13,394 & 5,853 & $5,853(43.6 \%)$ \\
DBP-map & 1,127 & 898 & $898(79.6 \%)$ \\
WD-truthy & 3,346 & 1,922 & $1,067(31.8 \%)$ \\
Freebase & 8,289 & 1,723 & $1,687(20.3 \%)$ \\
\hline Total & 26,156 & 10,396 & $9,505(36.3 \%)$ \\
\hline
\end{tabular}

Table 7: Predicted counting predicates across different KBs, where Input is KB predicates (direct only), Output is from the classifier prediction and Filtered the number of predicates remaining after removing predicates related to IDs and codes.

b. Important Features. The most important features in the counting predicate classifier are the mean and 10th percentile of the count values of a predicate with negative weights of 0.006 and 0.031 suggesting that counting predicates usually take smaller integer values. The predicate domain of type Organization has a positive weight of 0.14 .

The determining features of the enumerating classifier are the type information on the predicate domain and range. For example, the weights for domain Thing and range Organization are positive values of 0.135 and 0.046 , respectively. It is interesting to note that predicate ranges of type Work and Place have small negative weights of 0.008 and 0.097 , respectively, suggesting that predicates with range type location are less likely to be enumerating predicates.

c. Predicted Set Predicates. The number of set predicates predicted by each classifier is shown in Tables 6 and 7 in the Output column. We have 10,396 predicted as counting predicates by the counting classifier out of 26,156 . The percentage of predicted counting predicates is almost $40 \%$ which is much higher than the class distribution in the training data $(11 \%)$. One reason is the very low precision scores of the classifiers which may lead to more false positives. The enumerating classifier predicts $12,366(34 \%)$ of 36,244 predicates as enumerating predicates which is closer to the class distribution seen in the training data (40\%).

We illustrate some predicted counting predicates in Table 8 and a few enumerating predicates in Table 9 The DBpedia raw KB predicate voiceActor ${ }^{-1}$, for example, connects a voice actor to the associated show 8 and

${ }^{8}$ List of shows Mel Blanc voiced over https://tinyurl.com/ dbpedia-mel-blanc

\begin{tabular}{|clr|}
\hline KB & Counting Predicates \\
\hline DBP-raw & $\begin{array}{l}\text { employees, retiredNumbers, } \\
\text { crewMembers, } \\
\text { members }\end{array}$ \\
DBP-map & $\begin{array}{l}\text { numberOfStudents, facultySize, } \\
\text { numberOfGoals, populationAs0f, } \\
\text { capacity } \\
\text { employees, } \\
\text { numberOfConstituencies, } \\
\text { numberOfSeats } \\
\text { children, } \\
\text { population, } \\
\text { injuries, passengers }\end{array}$ \\
FB & Wrong Predictions \\
\hline DBP-raw & $\begin{array}{l}\text { linecolor, km, birthyear, } \\
\text { foundingYear, keyPerson } \\
\text { publicationDate, } \\
\text { coordinateLocation }\end{array}$ \\
WD-truthy & $\begin{array}{l}\text { maxLength, height } \\
\text { FB }\end{array}$
\end{tabular}

Table 8: Example predicted counting predicates from the different KBs.

employees 9 gives the number of employees in an organization.

The classifiers also misclassify as shown in previous tables, for example, the counting classifer wrongly predicts dates like birthYear and foundingYear, measurements such as $\mathrm{km}$, height as counting predicates. The enumerating classifier makes errors by positively labelling functional and pseudo-functional predicates like currentTeam, sourceOf Income.

d. Filtering identifier labels. Our classifiers, especially the counting classifier has lower precision than recall. One of the commonly occurring type of predicates are identifiers, which may be represented as a fact with an large number in integer or string format and, we can remove such predicates without losing any actual set predicate. The filtering is done by checking for the presence of the words ' $i d$ ' and 'code' as substrings, but not part of a longer word, in the predicate label, irrespective of the source KB. In Table 10 we compare the number of identifier predicates that need to be filtered before classification versus the number of predicates filtered after classification. The enumerating classifier is good at filtering identifier predicates since almost $90 \%$ of the identifier labels are predicted to be false. The counting classifier removes around $59 \%$ of the identifier predicates and could benefit from the identifier filter. Thus we apply the identifier label filter only on the output of the classifiers, and the final numbers are shown in the Filtered column of Tables 6 and 7 .

e. Statistical alignment. The $N D C G$ scores reported in Table 11 are an evaluation of the top three alignments

${ }^{9}$ Sample of subjects with the predicate employees https:// tinyurl.com/dbpedia-employees 


\begin{tabular}{|c|c|}
\hline KB & Enumerating Predicates \\
\hline DBP-raw & $\begin{array}{r}\text { workInstitution, } \\
\text { members }^{-1}, \\
\text { nativeLangugae }\end{array}$ \\
\hline DBP-map & $\begin{array}{l}\text { recordLabel, developer, product, } \\
\text { publisher, formerCoach }{ }^{-1} \text { employer } \\
\text { governor }\end{array}$ \\
\hline WD-truthy & $\begin{array}{l}\text { participantOf }{ }^{-1}, \quad \text { airlineHub, } \\
\text { developer, father }\end{array}$ \\
\hline FB & $\begin{array}{l}\text { actor, member, starring, publisher, } \\
\text { airportsServed }^{-1}, \text { foundedLocation }^{-1}\end{array}$ \\
\hline \multicolumn{2}{|r|}{ Wrong Predictions } \\
\hline DBP-raw & currentTeam, deathCause, weightClass \\
\hline DBP-map & secondTeam, genre \\
\hline $\begin{array}{l}\text { WD-truthy } \\
\text { FB }\end{array}$ & $\begin{array}{l}\text { parentOrganization, hairColor } \\
\text { cameras, burstCapability, founder }\end{array}$ \\
\hline
\end{tabular}

Table 9: Example predicted enumerating predicates from the different KBs.

\begin{tabular}{|c|cc|c|}
\hline Class & Pre-filter & Post-filter & Classifier \\
\hline Enumerating & 2,167 & 151 & $2,016(93 \%)$ \\
Counting & 2,158 & 881 & $1,277(59 \%)$ \\
\hline
\end{tabular}

Table 10: The number of identifier predicates present in the input to the classifiers Pre-filter vs. the number present in the predicted predicates Post-filter successfully removed by the classifiers.

from all nine alignment metrics based on relevance judgments collected from crowd workers. We report the $N D C G$ at positions 1 and 3 . The table is divided into the three alignment families and we consider two directions. The first is the direction from a counting predicate to its enumerating predicate alignments and the second is the reverse.

Based on the scores presented in Table 11, we can conclude that the linguistic similarity metric of cosine similarity (defined in Sec. 6C) performs the best individually, except for NDCG@3 for the counting to enumerating direction, where the Pearson correlation measure performs best. The Correlation metric in the counting to enumerating direction and the P'fect $M R$ metric in the reverse direction are the best performing metrics of the set predicate value distribution family (defined in Sec. 6B). The strongest metrics in the set predicate co-occurrence family (defined in Sec. 6A) are Conditional $_{E}$ in the direction of counting to enumerating predicate alignment and $P^{\prime}$ wise $M I$ in the other direction.

The Combined metric takes the best performing metric from each family and computes the mean of the alignment scores to obtain a combined score which gives better results than any individual metric. We use this combined measure to rank our alignments.

f. Transferability. We also evaluate the transferability of set predicate identification models trained on one $\mathrm{KB}$, evaluated on the others. We do this for all combinations

\begin{tabular}{|l|cc|cc|}
\hline \multirow{2}{*}{ Metric } & \multicolumn{2}{|c|}{ Counting } & \multicolumn{2}{c|}{ Enumerating } \\
& @1 & $@ 3$ & @1 & $@ 3$ \\
\hline Absolute & 0.71 & 0.56 & 0.62 & 0.63 \\
Jaccard $_{\text {Conditional }_{C}}$ & 0.76 & 0.61 & 0.69 & 0.67 \\
Conditional $_{E}$ & 0.76 & 0.56 & 0.68 & 0.67 \\
P'wiseMI $^{\prime}$ & 0.73 & 0.58 & 0.71 & 0.70 \\
\hline P'fectMR $^{\prime}$ Correlation & 0.70 & 0.57 & 0.73 & 0.72 \\
P'tileVM $^{\prime}$ & 0.77 & 0.69 & 0.62 & 0.61 \\
\hline CosineSim $^{\text {Combined }}$ & 0.79 & 0.57 & 0.65 & 0.65 \\
\hline Com4 & $\mathbf{0 . 8 4}$ & $\mathbf{0 . 6 7}$ & $\mathbf{0 . 7 5}$ & $\mathbf{0 . 7 5}$ \\
\hline
\end{tabular}

Table 11: Average $N D C G$ scores for the alignment stage.

\begin{tabular}{|l|cccc|}
\hline & DBP-raw & DBP-map & WD-truthy & FB \\
\hline DBP-raw & 57.1 & 36.0 & 17.6 & - \\
DBP-map & 58.5 & 52.5 & 50.0 & - \\
WD-truthy & 54.7 & 51.4 & 52.0 & - \\
FB & 41.4 & 41.8 & 8.5 & 80.0 \\
Random & 39.4 & 33.3 & 33.3 & 60.8 \\
\hline
\end{tabular}

Table 12: F1 scores for enumerating predicate classifiers, where rows heads represent the training sets and the column heads represent the test sets.

of KBs and report the F1 scores for the enumerating predicates in Table 12 and the counting predicates in Table 13. In each setting we also report the F1 scores of a random baseline.

We observe that in most cases, the classifiers significantly outperform the random baseline, although the performance is quite low when evaluating on the Wikidatatruthy and Freebase KBs. The task of predicting enumerating predicates in the two variants of DBpedia is better when trained on any of the remaining KBs, whereas no classifier can predict enumerating predicates in Freebase. In the counting predicate prediction, the classifier trained on DBpedia-map performs worse than random classifier for all test data. Here too, the performance of classifiers on Freebase is quite low. In contrast, the classifiers trained on Freebase perform well on DBpedia in both cases. We can conclude that the case of counting predicate prediction is more challenging, given that the F1 scores of the random classifier and the training data are lower. Additionally, training on a single $\mathrm{KB}$ does not fare well in the case of counting predicate prediction.

\section{Use Cases}

Question answering. Question answering benefits from set predicate alignment at three stages: (i) natural language parsing, (ii) query result debugging, (iii) query result enrichment.

In natural language parsing, state-of-the-art KBQA systems typically generate a set of candidate parses, which subsequently are ranked, and the top one executed (with research prototypes often allowing the user to inspect and 


\begin{tabular}{|l|cccc|}
\hline & DBP-raw & DBP-map & WD-truthy & FB \\
\hline DBP-raw & 56.5 & 5.2 & 33.3 & - \\
DBP-map & 15.1 & 43.7 & 11.7 & 12.1 \\
WD-truthy & 25.9 & 20.4 & 32.0 & 8.5 \\
FB & 34.6 & 13.7 & 7.4 & 40.0 \\
Random & 18.7 & 11.1 & 14.2 & 14.2 \\
\hline
\end{tabular}

Table 13: F1 scores for counting predicate classifiers, where rows heads represent the training sets and the column heads represent the test sets.

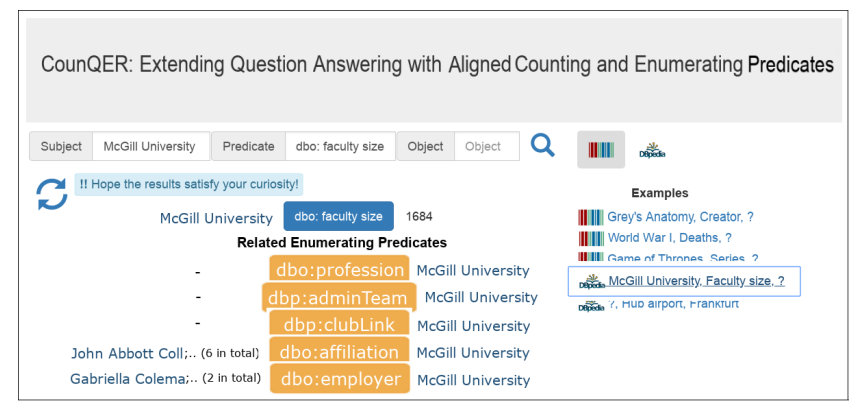

Figure 3: Demo system for question answering.

choose among them). Set predicate alignments could be used to generate further candidates, or used as ranking feature.

Structured queries over KBs often produce empty or otherwise unexpected results that require query debugging. Set predicate alignments could help to understand whether an ambiguous predicate name refers to the intended predicate, and related counts could help to understand that a predicate is an intended one, but that just the $\mathrm{KB}$ is incomplete.

Finally, in the absence of any problems, results from aligned predicates can enhance the user experience and anticipate follow-up information needs. Given a query such as "SELECT ?val WHERE \{dbp:McGill_University dbo:faculty_size ?val. $\}$ ", we can, in addition to the actual count, directly provide example instance (see Fig. 3) 10 Conversely, if the user asks for people working at McGill University, we can, in addition to instances, provide (an estimate of) the total count.

We are in the process of building a demo for that purpose. In the demo interface, users can input a single-triple Wikidata or DBpedia query by specifying a predicate and a subject or an object. Along with the results for the other field, the interface will then show a ranked list of aligned set predicates, both, those having values (bottom 2 in Fig. 3), and those having no values (top 3 in same Figure). This makes the demo relevant for two use cases: (i) KB curation, by checking which related predicates have missing information so far, and (ii) question answering, by enhancing count questions with instance information, and vice versa. A video of the demo prototype can be found at

\footnotetext{
${ }^{10}$ Google Web Search shows this behaviour for queries such as "number of movies by Tarantino".
}

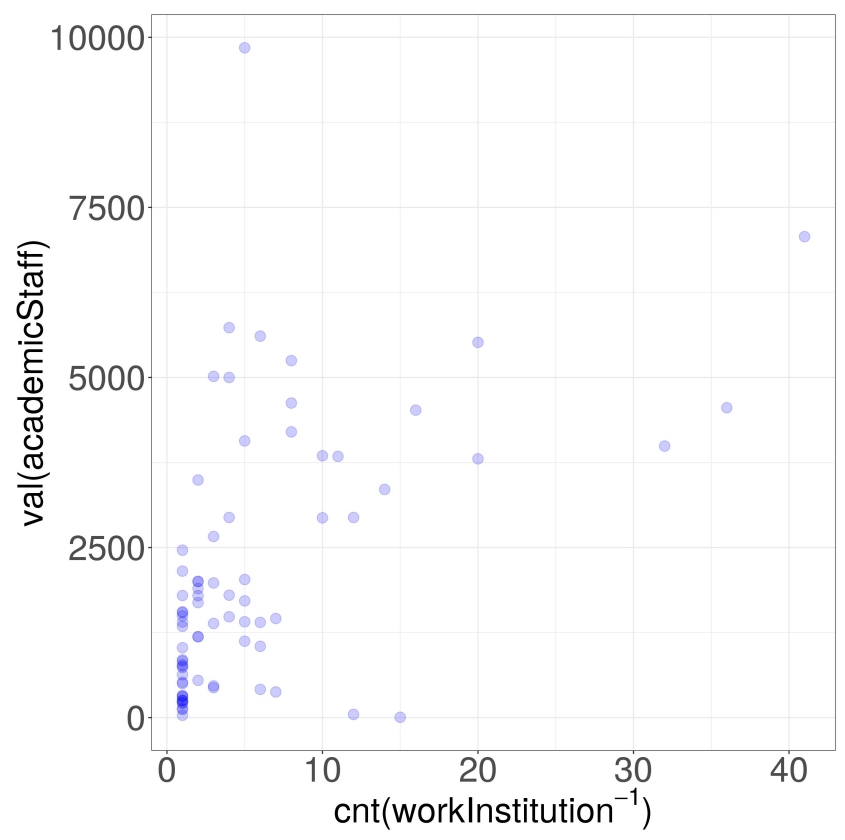

Figure 4: Value distribution of academicStaff and count of workInstitution ${ }^{-1}$ across 76 subjects in DBpedia raw KB.

https://tinyurl.com/y2ka4kfu, and the demo can be accessed at https://counqer.mpi-inf .mpg.de/spo.

KB curation. In this section we look into a few alignments from different $\mathrm{KBs}$ and the distribution of their values. The first alignment in Fig. 4 is the pair (work Institution $^{-1}$, academicStaff) from the DBpedia raw $\mathrm{KB}$, which co-occurs across 76 subjects (institutions). Each point $(x, y)$ in the plot represents an institution, which is connected to $x$ entities by the predicate workInstitution ${ }^{-1}$, and which takes the value y for the predicate academicStaff. In an ideal condition the count of instances should match the value and all points should lie along the line $y=x$. Points lying above this line suggest incompleteness. Such is the case in Fig. 4 where the predicate workInstitution ${ }^{-1}$ is often only connected to the popular or important staff members.

Next we look into an alignment from DBpedia mapping based KB, (employer ${ }^{-1}$, numberOfEmployees) in Fig. 5. In this alignment we also observe that the enumerated facts is much smaller than the number of employees, typically because such facts only exist for the most important employees.

In Fig. 6, we show an alignment from the Wikidata $\mathrm{KB}$ which is regarding the members of a political party (memberOfPoliticalParty ${ }^{-1}$, memberCount). Similar to the previous trends, here also the number of enumerated facts about the members in a political party is less than the actual value. The final alignment we show is of the pair (placeOfBirth ${ }^{-1}$, populationState) in the Freebase KB as shown in Fig. 7. From the numbers it seems that the predicate covers small geographical locations where the number of enumerated facts is more complete than in 


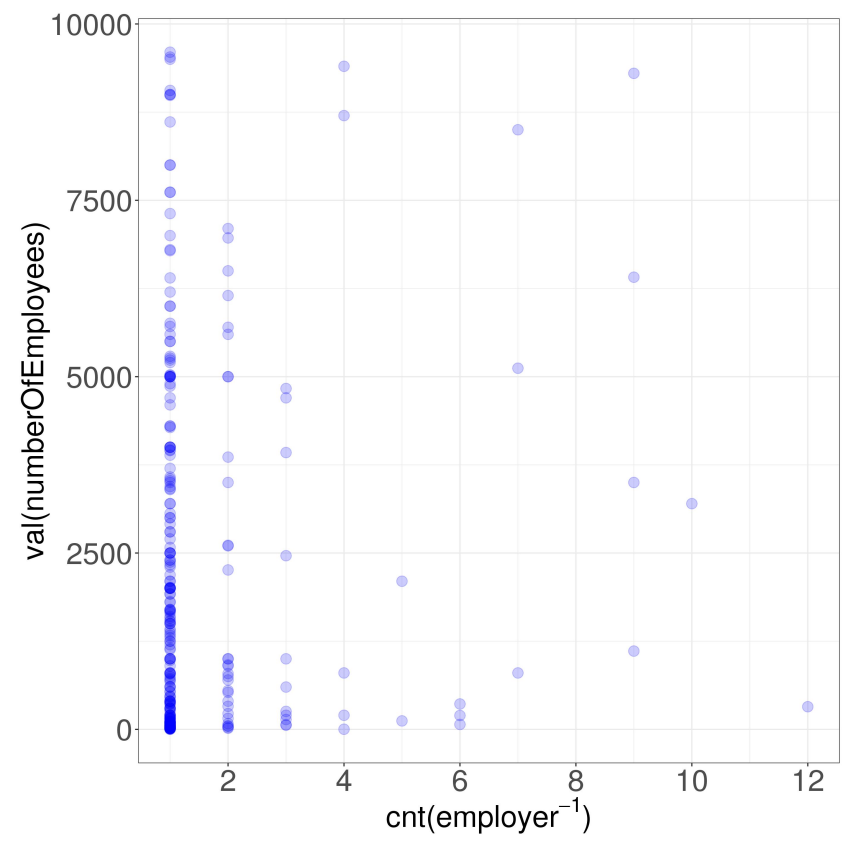

Figure 5: Value distribution of counting predicate number 0 Employees and count of enumerating predicate employer ${ }^{-1}$ across 278 subjects in DBpedia mapping-based KB.

the previous cases.

In each of the alignment figures there are at most two instances where the value of the counting predicate is less than the count of enumerated instances, there exist no such instances in the Fig. 7. Such a low number of points indicate anomaly or inconsistencies such as a backdated counting predicate value or, for instance, in Fig. 5 where the value of counting predicate numberofEmployees is 0 even though there exist enumerated facts.

Figure 8 shows the value distribution of an alignment where each cell in position $(x, y)$ gives the number of subjects which takes $x$ as the value of the counting predicate and $y$ number of instances with the enumerating predicate. The first analysis concerns the places where a sports event took place. A notable anomaly in DBpedia raw KB is that regularly, for each venue, both the stadium and the city were recorded. Thus, we plot two green lines showing 1:1 matches, and 2:1 matches. Instances below both lines likely point to incompleteness (some stadiums are missing), instances below both lines likely point to some errors in the data (i.e., too many stadiums added). As one can see, the completeness appears to be relatively high, while there are several cases that deserve closer inspection w.r.t. possible incorrectness.

The second analysis concerns the number of members of local councils, compared with individual members listed. Here incompleteness is prevalent, with typically only 1 in 30 to 1 in 10 members listed.

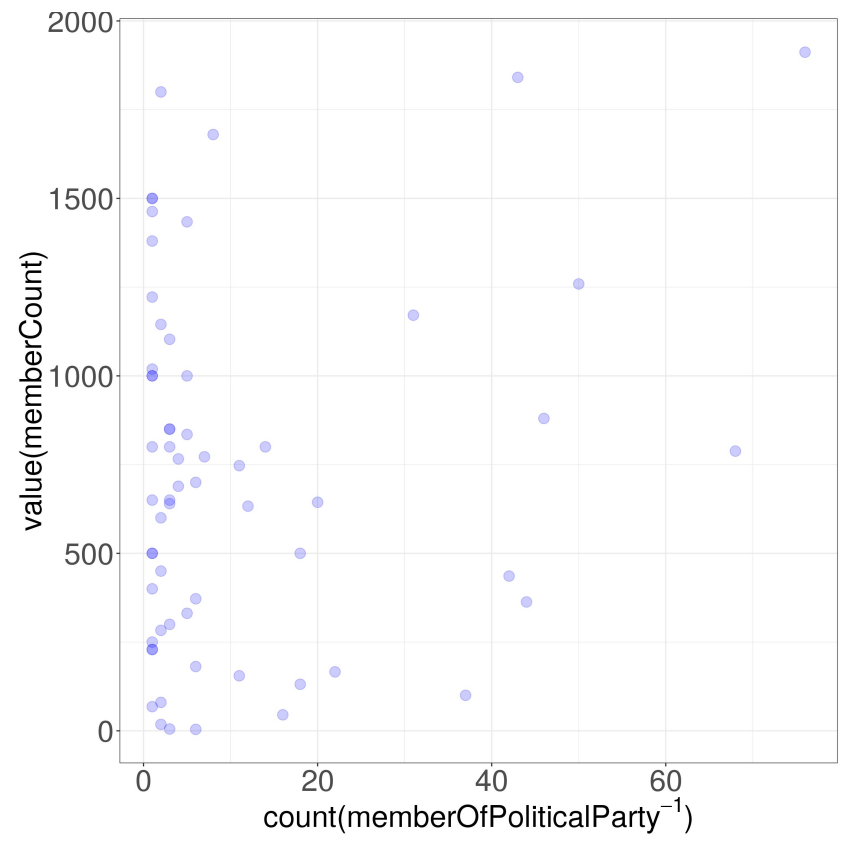

Figure 6: Value distribution of counting predicate memberCount and count of enumerating predicate member0fPoliticalParty ${ }^{-1}$ across 62 subjects in Wikidata-truthy KB.

\section{Discussion}

Limitations and usage implications. While the experimental results are encouraging, F1-scores of $35-55 \%$ for the identification stage indicate that there is still much room for improvement. Further feature engineering, e.g., regarding IDs and codes, and further distribution measures, may still yield moderate improvements, but we see three principled challenges that limit any fully-automated approach: (i) statistical cues allow to rule out well some clear negatives, but for many infrequent predicates, provide only weak signals. (ii) textual cues for predicates are mostly short and thus of limited informativeness. (iii) input KBs come with a considerable level of noise.

Where possible, we would therefore recommend to execute set predicate identification and alignment not in a fully automated manner, but employ a human-in-the-loop process, where statistical procedures narrow the search space for human annotators, or human annotators focus on the "fat head" (e.g., the 100 most used predicates), and automated methods focus on the long tail. In industrial deployment of the question answering use case, human input could also come from user feedback, e.g., query-clicklogs or query reformulations and follow-up questions.

Inverse predicates. For generalizability, our method currently does not incorporate existing definitions of inverse predicates. Freebase contains $12 \mathrm{~K}$ such definitions (via owl: inverse0f [9]), Wikidata has 136 (identified via the meta-property inverse property), DBpedia has three mentions in comment fields. Incorporating such KB-specific constraints could further boost the accuracy of alignments. 


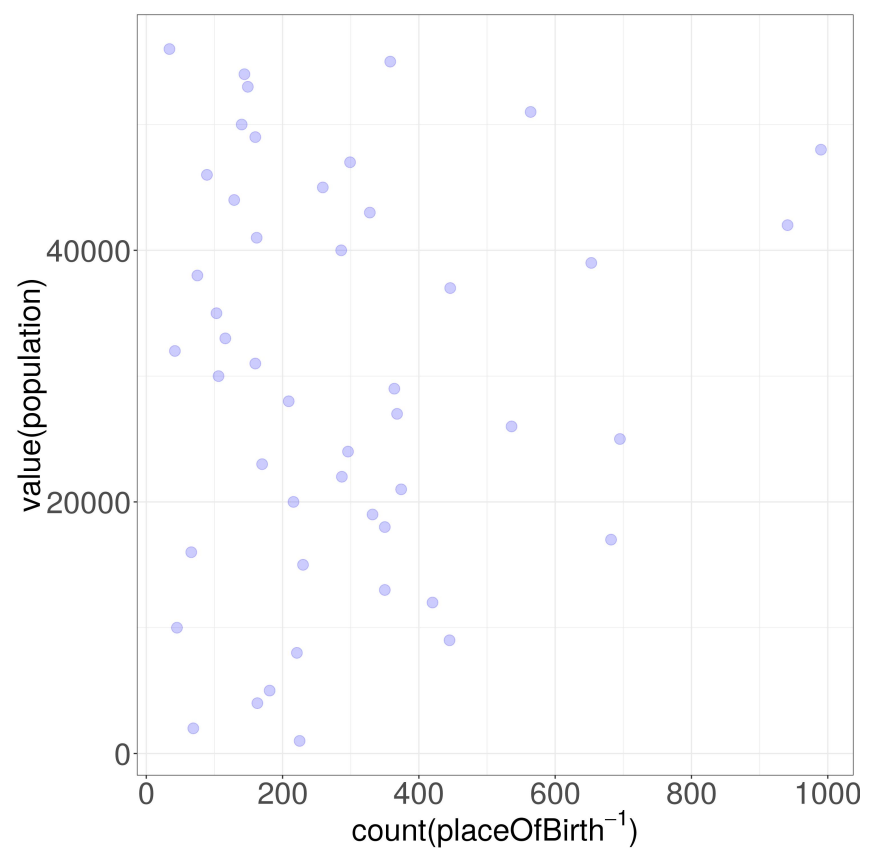

Figure 7: Value distribution of counting predicate populationState and count of enumerating predicate placeOfBirth ${ }^{-1}$ across 48 subjects in Freebase KB.

Transferability of method. A crucial aspect for our framework is whether it can be utilized on new KBs without requiring too much adaptation. Our modular framework is aimed towards this purpose. The supervised predicate classification stage allows to transfer our approach by only creating new training instances. For KBs where textual predicates are unavailable, a sensible extension is the incorporation of latent representations of predicates [48, 25. as separate features in the classification stage and by considering cosine similarity of predicate embeddings as a heuristic in the predicate alignment.

Indirect alignments. The alignments are also helpful in identifying redundancies in schema, where two or more set predicates (enumerating/counting) describing the same concept exist and are all aligned to a single set predicate of the other variant. For example the enumerating predicate affiliation in the DBpedia mapping-based KB aligns with the counting predicates ffacultySize, staff, numberOfStaff\}.

Multi-hop alignments. Counting predicates may well align with multi-hop paths of enumerating predicates. For instance, an interesting near-subset of population ( $x, y)$ is worksAt $(y, z)$, basedIn $(z, x)$. The search space for such alignments would grow quadratically, but clever pruning may keep it manageable.

Crowd annotation costs. The cost of the annotating classifier training data is approximately $0.13 \$$ per task per judgment. For the alignment evaluation task the cost was almost $0.40 \$$ per task per judgment. Thus the average cost per task is $0.5(0.13 * 3+0.4 * 3) \approx 0.80 \$$ if we collect
3 judgments. Given the average time spent per task, we arrive at an hourly pay of $\$ 14$, which corresponds to the salary of student assistants in Germany.

Open information extraction. So far we have only considered the alignment of canonicalized KB predicates. An interesting direction would be to extend this alignment towards open information extraction and open knowledge bases in the style of Reverb [12], i.e., to align textual phrases like " $X$ has $Y$ employees" with phrases like " $Z$ works at $Y$ ", " $Z$ recently joined $X$ ", etc. Numeric open information extraction traditionally focuses on temporal information [26] and measures [39], though there are also some recent works on counting information extraction [29, 30, which one might build upon.

\section{Conclusion}

In this paper we have introduced the problem of set predicate alignment, and presented CounQER, a methodology for identifying and linking set predicates that combines co-occurrence, correlational and linguistic features. We have shown that automated methods can identify and align set predicates on four diverse knowledge bases, and that these alignments are useful for use cases in knowledge base curation and question answering.

We believe that understanding set predicate semantics in today's KBs is an important step towards a better interaction with structured world knowledge repositories. Our next goals are to extend this methodology to multi-hop alignments, and towards open predicate phrases extracted from natural language texts.

\section{References}

[1] Algergawy, A., et al.: Results of the ontology alignment evaluation initiative 2018. In: Ontology Matching workshop (2018)

[2] Auer, S., Bizer, C., Kobilarov, G., Lehmann, J., Cyganiak, R., Ives, Z.: DBpedia: A nucleus for a web of open data (ISWC 2007)

[3] Bast, H., Haussmann, E.: More accurate question answering on Freebase (CIKM 2015)

[4] Boldyrev, N., Spaniol, M., Weikum, G.: Multi-cultural interlinking of web taxonomies with ACROSS. Journal of Web Science (2018)

[5] Bollacker, K., Evans, C., Paritosh, P., Sturge, T., Taylor, J.: Freebase: a collaboratively created graph database for structuring human knowledge. In: SIGMOD (2008)

[6] Calvanese, D., Cogrel, B., Komla-Ebri, S., Kontchakov, R., Lanti, D., Rezk, M., Rodriguez-Muro, M., Xiao, G.: Ontop: Answering sparql queries over relational databases. SWJ (2017)

[7] Calvanese, D., Eiter, T., Ortiz, M.: Regular path queries in expressive description logics with nominals (AAAI 2009)

[8] Calvanese, D., Lenzerini, M., Nardi, D.: Description logics for conceptual data modeling. In: Logics for databases and information systems (1998)

[9] Chah, N.: OK Google, what is your ontology? or: Exploring Freebase classification to understand Google's knowledge graph. arXiv preprint arXiv:1805.03885 (2018)

[10] Dell, H., Roth, M., Wellnitz, P.: Counting answers to existential questions. ICALP (2019)

[11] Euzenat, J., Shvaiko, P.: Ontology matching. Springer (2007) 


\begin{tabular}{|c|c|c|c|c|c|c|c|c|c|c|}
\hline & \multicolumn{9}{|c|}{ count(stadium) } \\
\hline & & 1 & 2 & 3 & 4 & 5 & 6 & 7 & 8 & $\geq 8$ \\
\hline \multirow{8}{*}{ 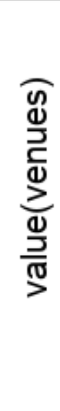 } & 1 & 290. & 420 & 59 & 32 & 3 & 7 & 3 & 1 & 8 \\
\hline & 2 & 14 & 971 & 121 & 167 & 22 & 13 & 3 & 1 & 4 \\
\hline & 3 & 4 & 3 & 11 & 29 & 21 & $48^{2}$ & 15 & 5 & T \\
\hline & 4 & 2 & 1 & 1 & & 9 & 9 & 19 & 50 & 14 \\
\hline & 5 & 1 & 2 & & 2 & 2 & 5 & 2 & 8 & 43 \\
\hline & 6 & 1 & 1 & inco & omple & & 6 & 6 & 3 & 38 \\
\hline & 8 & & & & 2 & & & 1 & & 18 \\
\hline & $=0$ & 2 & & 1 & 1 & & 1 & 2 & 4 & 47 \\
\hline
\end{tabular}

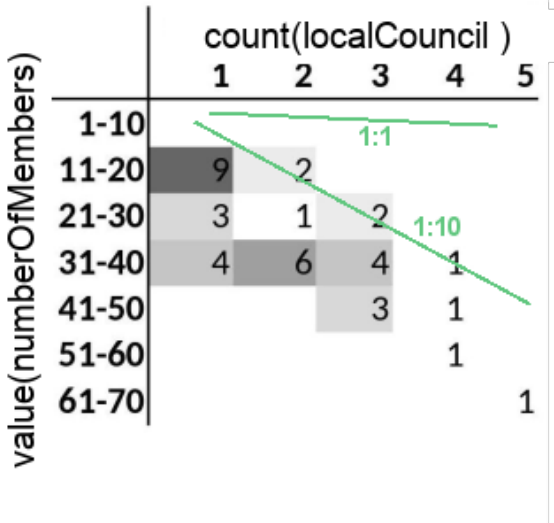

Figure 8: Value distribution of the counting predicate venues and count of enumerating predicate stadium for 2179 sports events (left), and numberOfMembers and count of localCouncil ${ }^{-1}$ for 35 political assemblies (right) from the DBpedia raw KB.

[12] Fader, A., Soderland, S., Etzioni, O.: Identifying relations for open information extraction (EMNLP 2011)

[13] Fan, W., Wu, Y., Xu, J.: Adding counting quantifiers to graph patterns (SIGMOD 2016)

[14] Galárraga, L., Razniewski, S., Amarilli, A., Suchanek, F.M.: Predicting completeness in knowledge bases. In: WSDM (2017)

[15] Galárraga, L.A., Teflioudi, C., Hose, K., Suchanek, F.: AMIE: association rule mining under incomplete evidence in ontological knowledge bases. In: WWW (2013)

[16] Gelman, A., Jakulin, A., Pittau, M.G., Su, Y.S., et al.: A weakly informative default prior distribution for logistic and other regression models. The Annals of Applied Statistics (2008)

[17] Glimm, B., Lutz, C., Horrocks, I., Sattler, U.: Conjunctive query answering for the description logic SHIQ. JAIR (2008)

[18] Hollunder, B., Baader, F.: Qualifying number restrictions in concept languages. KR (1991)

[19] Hopkinson, A., Gurdasani, A., Palfrey, D., Mittal, A.: DemandWeighted Completeness Prediction for a Knowledge Base. NAACL (2018)

[20] Jain, P., Hitzler, P., Sheth, A.P., Verma, K., Yeh, P.Z.: Ontology alignment for linked open data (ISWC 2010)

[21] Järvelin, K., Kekäläinen, J.: Cumulated gain-based evaluation of IR techniques. TOIS (2002)

[22] Koutraki, M., Preda, N., Vodislav, D.: Online relation alignment for linked datasets. In: ESWC (2017)

[23] Lehmann, J., Hitzler, P.: Concept learning in description logics using refinement operators. Machine Learning (2010)

24] Lehmann, J., Isele, R., et al.: DBpedia-a large-scale, multilingual knowledge base extracted from Wikipedia. SWJ (2015)

[25] Lin, Y., Liu, Z., Sun, M., Liu, Y., Zhu, X.: Learning entity and relation embeddings for knowledge graph completion. In: AAAI (2015)

[26] Ling, X., Weld, D.S.: Temporal information extraction (AAAI 2010)

[27] Luggen, M., Difallah, D., Sarasua, C., Demartini, G., CudréMauroux, P.: Non-parametric class completeness estimators for collaborative knowledge graphsthe case of wikidata. In: ISWC (2019)

[28] McGuinness, D.L., Van Harmelen, F., et al.: OWL web ontology language overview. W3C recommendation (2004)

[29] Mirza, P., Razniewski, S., Darari, F., Weikum, G.: Cardinal virtues: Extracting relation cardinalities from text (ACL 2017)

[30] Mirza, P., Razniewski, S., Darari, F., Weikum, G.: Enriching knowledge bases with counting quantifiers (ISWC 2018)

[31] Neumaier, S., Umbrich, J., Parreira, J.X., Polleres, A.: Multilevel semantic labelling of numerical values (ISWC 2016)

[32] Niepert, M., Meilicke, C., Stuckenschmidt, H.: A probabilisticlogical framework for ontology matching (AAAI 2010)
[33] Nikolaou, C., Kostylev, E.V., Konstantinidis, G., Kaminski, M., Grau, B.C., Horrocks, I.: Foundations of ontology-based data access under bag semantics. AI (2019)

[34] Paulheim, H.: Knowledge graph refinement: A survey of approaches and evaluation methods. SWJ (2017)

[35] Pennington, J., Socher, R., Manning, C.D.: Glove: Global vectors for word representation (EMNLP 2014)

[36] Rahm, E., Bernstein, P.A.: A survey of approaches to automatic schema matching. VLDB Journal (2001)

[37] Razniewski, S., Jain, N., Mirza, P., Weikum, G.: Coverage of information extraction from sentences and paragraphs. In: EMNLP (2019)

[38] Razniewski, S., Suchanek, F., Nutt, W.: But what do we actually know? In: AKBC Workshop (2016)

[39] Saha, S., Pal, H., et al.: Bootstrapping for numerical Open IE (ACL 2017)

[40] Shvaiko, P., Euzenat, J.: Ontology matching: state of the art and future challenges. TKDE (2013)

[41] Shvaiko, P., Euzenat, J., Jiménez-Ruiz, E., Cheatham, M., Hassanzadeh, O. (eds.): Ontology Matching workshop. CEUR-WS (2018)

[42] Soulet, A., Giacometti, A., Markhoff, B., Suchanek, F.M.: Representativeness of knowledge bases with the generalized benfords law. In: ISWC (2018)

[43] Subercaze, J.: Chaudron: extending DBpedia with measurement (ESWC 2017)

[44] Suchanek, F.M., Abiteboul, S., Senellart, P.: PARIS: Probabilistic alignment of relations, instances, and schema. VLDB (2011)

[45] Suchanek, F.M., Kasneci, G., Weikum, G.: YAGO: a core of semantic knowledge (WWW 2007)

[46] Tibshirani, R.: Regression shrinkage and selection via the lasso. Journal of the Royal Statistical Society (1996)

[47] Vrandečić, D.: Wikidata: A new platform for collaborative data collection (WWW 2012)

[48] Wang, Z., Zhang, J., Feng, J., Chen, Z.: Knowledge graph embedding by translating on hyperplanes. In: AAAI (2014)

[49] Wang, Z., Li, J., Zhao, Y., Setchi, R., Tang, J.: A unified approach to matching semantic data on the web. KnowledgeBased Systems (2013)

[50] Wienand, D., Paulheim, H.: Detecting incorrect numerical data in DBpedia (ESWC 2014)

[51] Wu, H., Villazon-Terrazas, B., Pan, J.Z., Gomez-Perez, J.M.: How redundant is it?: An empirical analysis on linked datasets. COLD (2014)

[52] Zaveri, A., Rula, A., Maurino, A., Pietrobon, R., Lehmann, J., Auer, S.: Quality assessment for linked data: A survey. SWJ (2016) 